From the Departments of Hematology and Gynecology, University Medical Centre Groningen, University of Groningen, Groningen; Department of Hematology, University Medical Centre Nijmegen, Nijmegen; Department of Radiotherapy, Leiden University Medical Centre, Leiden, the Netherlands; the Clinical Research Unit; Groupe Régional d'Etudes sur le Cancer (GRECAN 1772), University of Caen Basse-Normandie, Centre François Baclesse, Caen Department of Hematology, Institut Bergonié, Bordeaux; the Department of Medical Oncology, Institut Gustave Roussy, Villejuif, France; and the Department of Oncology, UZ Gasthuisberg, Leuven, Belgium.

Submitted December 1, 2006; accepted April 4, 2007; published online ahead of print at www.jco.org on May 21, 2007

Supported by research grants from the Lance Armstrong Foundation.

Authors' disclosures of potential conflicts of interest and author contributions are found at the end of this article.

Address reprint requests to Hanneke C. Kluin-Nelemans, MD, PhD, Department of Hematology, University Medical Centre Groningen, University of Groningen, Hanzeplein 1, 9700 RB PO Box 30.001, Groningen, the Netherlands; e-mail: j.c.kluin@int.umcg.nl.

(C) 2007 by American Society of Clinical Oncology

0732-183X/07/2519-2825/\$20.00

DOI: $10.1200 / J C O .2006 \cdot 10.2020$

\title{
Gonadal Function in Males After Chemotherapy for Early- Stage Hodgkin's Lymphoma Treated in Four Subsequent Trials by the European Organisation for Research and Treatment of Cancer: EORTC Lymphoma Group and the Groupe d'Étude des Lymphomes de l'Adulte
}

Marleen A.E. van der Kaaij, Natacha Heutte, Nolwenn Le Stang, John M.M. Raemaekers, Arnold H.M. Simons, Patrice Carde, Evert M. Noordijk, Christophe Fermé, José Thomas, Houchingue Eghbali, Hanneke C. Kluin-Nelemans, and Michel Henry-Amar

$$
\begin{array}{llllllll}
\text { A } & \text { B } & \text { S } & \text { T } & \text { R } & \text { A } & \text { C } & \text { T }
\end{array}
$$

\section{Purpose}

To analyze fertility in male patients treated with various combinations of radiotherapy and chemotherapy, with or without alkylating agents, or with radiotherapy alone for Hodgkin's lymphoma.

\section{Patients and Methods}

Follicle-stimulating hormone (FSH) levels were measured in patients with early-stage upperdiaphragmatic disease enrolled in four European Organisation for Research and Treatment of Cancer (EORTC) trials (H6-H9). Median follow-up after therapy was 32 months. Patients with FSH measurement at least 12 months after end of treatment $(n=355)$ were selected to assess post-treatment fertility. Patients with FSH measurement 0 to 9 months after therapy $(n=349)$ were selected to analyze fertility recovery; of these, patients with elevated FSH (> $10 \mathrm{U} / \mathrm{L}$; $\mathrm{n}=101)$ were followed until recovery. Factors predictive for therapy-related infertility were assessed by logistic regression.

\section{Results}

The proportion of elevated FSH was 3\% and $8 \%$ in patients treated with radiotherapy only or with nonalkylating chemotherapy (doxorubicin, bleomycin, vinblastine, dacarbazine [ABVD], epirubicin, bleomycin, vinblastine, prednisone [EBVP]); it was 60\% $(P<.001)$ after chemotherapy containing alkylating agents (mechlorethamine, vincristine, procarbazine, prednisone [MOPP], MOPP/doxorubicin, bleomycin, vinblastine [ABV], bleomycin, etoposide, doxorubicin, cyclophosphamide, vincristine, procarbazine, prednisone [BEACOPP]). After a median time of 19 months, recovery of fertility occurred in $82 \%$ of patients treated without alkylating chemotherapy. This proportion was $30 \%$, statistically $(P<.001)$ lower in those treated with alkylating chemotherapy, and median time to recovery was 27 months. The post-treatment proportion of elevated FSH increased significantly $(P<.001)$ with the dose of alkylating chemotherapy administered, and recovery was less frequent and slower after higher doses. Age more than 50 years and stage II disease also contributed to poor outcome.

\section{Conclusion}

Fertility can be secured after nonalkylating chemotherapy for Hodgkin's lymphoma. In contrast, alkylating chemotherapy has a dismal effect, even after a limited number of cycles.

\section{J Clin Oncol 25:2825-2832. (C) 2007 by American Society of Clinical Oncology}

\section{INTRODUCTION}

In Hodgkin's lymphoma (HL), the combination of high cure rates (80\% to $90 \%)$ and increasing incidence in patients aged 20 to 44 years have led to a significant number of patients with a wish to start or complete a family after cure of HL. ${ }^{1-3}$

Male fertility after therapy for HL has been studied. ${ }^{4-6}$ However, most studies consisted of small series. The most informative results come from three publications in which a dose-dependent relationship between the number of cycles of MOPP (mechlorethamine, vincristine, procarbazine, prednisone) chemotherapy and toxicity was demonstrated. $^{7-9}$ However, MOPP chemotherapy is no longer considered the best therapy for $\mathrm{HL}$ and has been replaced by newer regimens. ${ }^{10}$ Because most studies focused on MOPP-like 
chemotherapy regimens, pooling of data, larger series, and comparison with more recent and less toxic chemotherapy schedules is necessary.

The European Organisation for Research and Treatment of Cancer (EORTC) had prospectively collected data on complications after treatment for HL since the start of the H6 trial in 1982. These data offered the opportunity for an analysis of male fertility after five combined chemoradiotherapy regimens applied between 1982 and 2004 . Moreover, the size of the data set made possible analyses on the influence of various factors such as age, disease extension, presence of "B" symptoms, and treatment type on the preservation of gonadal function in males.

\section{PATIENTS AND METHODS}

\section{Patients}

A total of 2,362 male patients from 11 European countries were included in four consecutive randomized clinical trials between August 1982 and May 2004. Protocols and informed consent were submitted to and approved by local ethical committees. The studies were carried out in accordance with the Helsinki Declaration. Patients were aged 15 to 70 years and presented with histologically proven, previously untreated, classical, clinical stage I or II supradiaphragmatic HL with WHO performance status grade 0 to 2. Patients with concomitant or previous malignancies were excluded, as were patients with serious comorbidity, those with progressive disease during treatment, those who had received total nodal irradiation or more than one chemotherapy regimen, and patients for whom information about the treatment actually administered was missing. After exclusions, 2,064 male patients were suitable for analysis (Fig 1).

\section{Study Design: Randomized H6-H9 Trials}

Patients in the H6 trial (1982-1988) received either mantle-field radiotherapy, mantle-field and para-aortic radiotherapy, $3 \times \mathrm{MOPP}-$ mantle field radiotherapy $-3 \times$ MOPP, or $3 \times$ ABVD (doxorubicin, bleomycin, vinblastine, dacarbazine)-mantle field radiotherapy $-3 \times$ ABVD. ${ }^{11-14}$ Patients in the H7 trial (1988-1993) received either subtotal nodal irradiation (STNI) or six cycles of EBVP (epirubicin, bleomycin, vinblastine, prednisone) followed by involved-field radiotherapy (IF-RT) or six cycles of MOPP/ABV hybrid (mechlorethamine, vincristine, procarbazine, prednisone/doxorubicin, bleomycin, vinblastine) and IF-RT. ${ }^{15-17}$ The H8 trial (1993-1998) was a cooperative trial with the Groupe d'Étude des Lymphomes de l'Adulte (GELA). Patients received either STNI or three, four, or six cycles of MOPP/ABV hybrid and IF-RT or four cycles of MOPP/ABV and STNI. ${ }^{18}$ In the H9 trial (19982004), also in cooperation with the GELA, patients received either six cycles of EBVP with various doses of IF-RT or four to six cycles of ABVD and IF-RT or four cycles of BEACOPP baseline (cyclophosphamide, doxorubicin, vincristine, bleomycin, etoposide, procarbazine, prednisone) and IF-RT. ${ }^{19-22}$

Before start of treatment, data collected concerned patient characteristics, initial work-up for staging of the disease, and initial work-up for further evaluation of toxicity. After treatment, late toxicity measurements were performed at completion of initial treatment and at 1,2,3, and 5 years thereafter for the $\mathrm{H} 6$ trial; at completion of initial treatment and yearly thereafter for the $\mathrm{H} 7$ trial; and at completion of initial treatment, 2 and 5 years after completion of therapy, and every 5 years thereafter for the $\mathrm{H} 8$ and $\mathrm{H} 9$ trials. Additional toxicity evaluations could occur in particular during the first year after therapy. Late toxicity examinations for gonadal toxicity consisted of measurement of several hormonal parameters: follicle-stimulating hormone (FSH), luteinizing hormone (LH), and testosterone. In a limited number of cases, a spermquality examination evaluating sperm count, motility, and morphology was also performed.

\section{Analysis of Fertility}

Male fertility depends on the process of spermatogenesis. This process is supported and controlled by the Sertoli cells in the testis. Sertoli cells maintain a feedback loop with the adenohypophysis, which produces FSH to control their function. As such, FSH levels can be seen as a reflection of Sertoli cell function and spermatogenesis with an inverse relationship between the two. Because data from sperm analysis were often lacking, FSH was used in this study as the sole parameter to evaluate male fertility.

FSH was considered elevated, and thus indicative of damage to fertility, at more than $10 \mathrm{U} / \mathrm{L}$. Because data were issued from more than 60 laboratories from 11 countries within a time frame of more than 20 years, some safeguards were introduced. FSH values were dichotomized (normal/elevated): changes from one category to the other were checked blindly (without information on therapy) to state their significance. Small elevations crossing the upper level were ignored if the relative change was less than $20 \%$. Dichotomized values were also evaluated in relation to normal FSH values for the laboratory (provided for $90 \%$ of the patients) and a conclusion made using the same approach. When no information on normal values was available, the relatively high (and safe) cutoff point of $10 \mathrm{U} / \mathrm{L}$ was used.

Patients were selected provided that an FSH evaluation was available at least 1 year after the end of treatment. This selection enabled comparison with the results of published studies. An FSH evaluation before treatment was not available for all patients. To use these cases despite the lack of initial data, we first analyzed patients with pretreatment data and found elevated FSH levels in only $4 \%$ (11 of 294). Elevation ranged from 11.8 to $44 \mathrm{U} / \mathrm{L}$ with a median of $19.9 \mathrm{U} / \mathrm{L}$ (normal < $10 \mathrm{U} / \mathrm{L}$ ). These cases were excluded from further analysis. Next, when analyzing the follow-up of these 11 patients, we observed that in all three patients who had received nonalkylating chemotherapy, FSH levels normalized after therapy, underlining the probable lack of clinical significance of pretreatment elevated FSH. Therefore, we argued that both the very low percentage of patients with spontaneously elevated FSH and its presumable lack of clinical impact justified that patients without pretreatment FSH levels were included. Consequently, all patients without FSH evaluation before treatment were also included in the study. Overall, 355 patients fulfilled those criteria (Fig 1, Selection A).

In an attempt to study the change in FSH level with time after treatment, a second group was defined that included all patients with at least one FSH evaluation made during the 0 - to 9 -month period after treatment completion. The same assumption as above was applied to this group that consisted of 349 patients (Fig 1, Selection B). Of these, 203 had an FSH evaluation before treatment with a median value of $3.8 \mathrm{U} / \mathrm{L}$ (range, 0 to $10.1 \mathrm{U} / \mathrm{L}$ ).

\section{Statistical Analysis}

The aim of the study was to evaluate the hormonal fertility parameters of male patients after completion of radiotherapy alone or various combinations of chemotherapy and radiotherapy with or without alkylating agents. The proportion of elevated FSH after treatment and the time to fertility recovery were the main end points analyzed. Ninety-five percent CIs for the rate were estimated assuming a binominal distribution for the number of elevated FSH. Patients who received chemotherapy were grouped into those who had received regimens without alkylating agents (ABVD, EBVP) and those treated with alkylating chemotherapy (MOPP, MOPP/ABV, BEACOPP). The number of cycles of chemotherapy was used to estimate the amount of alkylating agents administered, counting one cycle of MOPP/ABV or BEACOPP as equivalent to 0.5 cycles of MOPP. Patient characteristics were compared using the Fisher's exact test or variance analysis as appropriate. Time to recovery of fertility was calculated from time of completion of initial planned therapy to time of first normal FSH value, time of last toxicity examination, or January 1, 2006, whichever came first. The cumulative probability of gonadal function recovery was calculated as [ 1 -the probability of surviving without normalization of FSH]. An analysis of prognostic factors with regard to fertility recovery was performed using Fisher's exact test (univariate analysis) and logistic regression (multivariate analysis). Factors analyzed were age $(<50 v \geq 50)$, stage (I $v$ II), and systemic (B) symptoms (absent $v$ present). Probability of fertility recovery was estimated according to the Kaplan-Meier method, and the rates between groups were compared with use of the log-rank test. Ninety-five percent CI for the rates were estimated using the method of Rothman and Boice. $^{23}$ Two-sided tests were used in reporting the results. Statistical significance was defined as a $P$ value of less than .05 . The SAS statistical software (release 9.1 TS Level 1M3; SAS Institute, Cary, NC) was used to analyze data. 


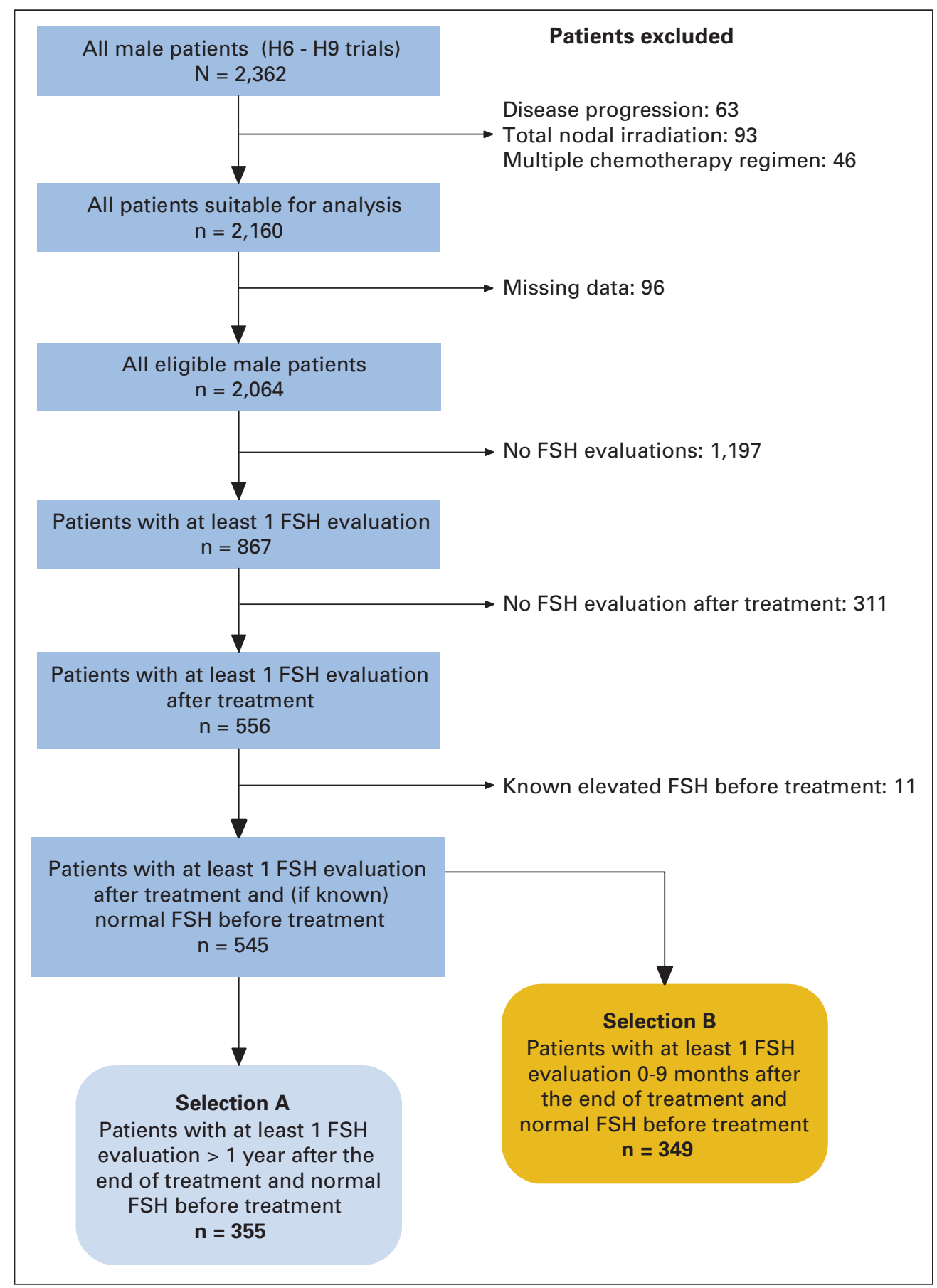

Fig 1. Selection of patients included in the fertility analysis. FSH, folliclestimulating hormone.

Data were selected from files stored at the Medical Research Unit, Centre François Baclesse, Caen, France. Data were updated on January 1, 2006.

\section{RESULTS}

Patient characteristics for all eligible patients $(\mathrm{N}=2,064)$ and those included in the two selected groups (Selection A, $\mathrm{n}=355$; Selection $\mathrm{B}$, $\mathrm{n}=349$ ) are listed in Table 1 . Overall, 174 patients (33\%) were selected in both groups for a total of 530 patients studied. Patients selected were more often issued from $\mathrm{H} 7$ and $\mathrm{H} 8$ trials in which toxicity evaluation was mandatory according to protocol. Age, clinical stage, and B-symptoms distributions were similar in the three groups. Treatments actually administered are indicated in Table 2. Radiotherapy alone was administered in one sixth of the patients, combined chemoradiotherapy without alkylating agents in one third, and a combination of radiotherapy and alkylating agents in more than one half.

\section{Effect of Therapy on Fertility (Selection A)}

In this group, time between end of treatment and FSH evaluation ranged from 12 to 135 months, with a median of 32 months. According to treatment groups, median time was 30 months for patients 


\begin{tabular}{|c|c|c|c|c|c|c|}
\hline \multirow[b]{2}{*}{ Characteristic } & \multicolumn{2}{|c|}{ Eligible Male Patients } & \multicolumn{2}{|c|}{$\begin{array}{c}>1 \text { Year After } \\
\text { Treatment (Selection A) }\end{array}$} & \multicolumn{2}{|c|}{$\begin{array}{c}\text { 0-9 Months After } \\
\text { Treatment (Selection B) }\end{array}$} \\
\hline & No. & $\%$ & No. & $\%$ & No. & $\%$ \\
\hline No. of patients & 2,064 & & 355 & 17 & 349 & 17 \\
\hline \multicolumn{7}{|l|}{ EORTC trial } \\
\hline H8 & 683 & 33 & 170 & 48 & 169 & 48 \\
\hline $\mathrm{H} 9$ & 719 & 35 & 20 & 6 & 54 & 16 \\
\hline \multicolumn{7}{|c|}{ Age at random assignment, years } \\
\hline Median & \multicolumn{2}{|c|}{32} & \multicolumn{2}{|c|}{30} & \multicolumn{2}{|c|}{31} \\
\hline Range & \multicolumn{2}{|c|}{$15-70$} & \multicolumn{2}{|c|}{$15-69$} & \multicolumn{2}{|c|}{$15-69$} \\
\hline$<30$ & 908 & 44 & 172 & 48 & 155 & 44 \\
\hline Unspecified & 14 & & 0 & & 1 & \\
\hline "B" symptoms present & 545 & 26 & 85 & 24 & 80 & 23 \\
\hline
\end{tabular}

treated with alkylating chemotherapy, 35 months for those treated with chemotherapy without alkylators, and 31 months for patients with radiotherapy only. FSH was elevated in $35 \%$ of patients (126 of 355 ). This proportion was $3 \%$ (two of $62 ; 95 \%$ CI, $0 \%$ to $11 \%$ ) in patients who received radiotherapy only, $8 \%$ (eight of $101 ; 95 \% \mathrm{CI}$, $3 \%$ to $15 \%$ ) in patients treated with chemotherapy without alkylators, and $60 \%$ (116 of $192 ; 95 \%$ CI, 53\% to $67 \%$ ) in patients treated with alkylating chemotherapy $(P<.001)$. The proportion of patients with post-treatment elevated FSH was also influenced by age $(P<.001)$, clinical stage $(P=.012)$, and the presence of B symptoms $(P=.003$; Table 3).

The amount of alkylating agents administered was estimated using the number of cycles administered and expressed in MOPP equivalents, counting one cycle of MOPP/ABV or BEACOPP as 0.5 MOPP equivalents. Forty-three patients received 1.5 MOPP equivalents, 67 patients 2.0 or $2.5 \mathrm{MOPP}$ equivalents and 82 patients three $(\mathrm{n}=64)$ or six $(\mathrm{n}=18)$ MOPP equivalents. The proportions of patients with post-treatment elevated FSH were 33\% (95\% CI, 19\% to

\begin{tabular}{|c|c|c|c|c|}
\hline \multirow[b]{2}{*}{ Treatment } & \multicolumn{2}{|c|}{ Selection A ( $n=355)$} & \multicolumn{2}{|c|}{ Selection B ( $n=349)$} \\
\hline & No. & $\%$ & No. & $\%$ \\
\hline Chemotherapy with alkylating agents & 192 & 54 & 185 & 53 \\
\hline Six cycles of MOPP + mantle-field RT & 18 & & $17^{*}$ & \\
\hline Three cycles of MOPP/ABV + IF-RT & 43 & & 47 & \\
\hline Four cycles of MOPP/ABV + IF-RT/STNI & 61 & & 56 & \\
\hline Six cycles of MOPP/ABV + IF-RT & $65+$ & & 56 & \\
\hline Four cycles of BEACOPP + IF-RT & 5 & & $9 \neq$ & \\
\hline Chemotherapy without alkylating agents & 101 & 28 & 107 & 31 \\
\hline Four cycles of ABVD + IF-RT & 7 & & 9 & \\
\hline Six cycles of ABVD + IF-RT/mantle-field RT & $21 \S$ & & 21 & \\
\hline Six cycles of EBVP \pm IF-RT & $73 \|$ & & 779 & \\
\hline Radiotherapy only & 62 & 18 & 57 & 16 \\
\hline IF-RT\# & 1 & & 2 & \\
\hline Subtotal nodal irradiation & 61 & & 55 & \\
\hline \multicolumn{5}{|c|}{$\begin{array}{l}\text { Abbreviations: MOPP, mechlorethamine, vincristine, procarbazine, prednisone; RT, ra } \\
\text { STNI, subtotal nodal irradiation; BEACOPP, bleomycin, etoposide, doxorubicin, c } \\
\text { bleomycin, vinblastine, prednisone. } \\
\text { "One patient received only five cycles. } \\
\text { tOne patient received only five cycles, one patient received STNI instead of IF-RT. } \\
\text { fOne patient received only two cycles. } \\
\text { §One patient received only five cycles. } \\
\text { \|One patient received only four cycles. } \\
\text { १One patient received only three cycles and STNI instead of IF-RT. } \\
\text { \#Major protocol violation, received only IF-RT, no chemotherapy. }\end{array}$} \\
\hline
\end{tabular}




\begin{tabular}{|c|c|c|c|c|c|}
\hline \multirow[b]{4}{*}{ Characteristic } & \multicolumn{5}{|c|}{$\begin{array}{l}\text { Table 3. Relationships Between Patient and Treatment Characteristics and Late } \\
\text { ( }>1 \text { year after the end of treatment) Change in FSH Level (Selection A, } n=355 \text { ) }\end{array}$} \\
\hline & \multicolumn{4}{|c|}{ FSH Level $>1$ Year After Treatment } & \multirow[b]{3}{*}{$P$} \\
\hline & \multicolumn{2}{|c|}{ Elevated } & \multicolumn{2}{|c|}{ Normal } & \\
\hline & No. & $\%$ & No. & $\%$ & \\
\hline Age at random assignment, years & & & & & $<.001$ \\
\hline$<30$ & 54 & 43 & 118 & 52 & \\
\hline$\geq 30$ and $<50$ & 49 & 39 & 101 & 44 & \\
\hline$\geq 50$ & 23 & 18 & 10 & 4 & \\
\hline \multicolumn{6}{|l|}{ Clinical stage } \\
\hline I & 29 & 23 & 83 & 36 & .012 \\
\hline ॥ & 97 & 77 & 146 & 64 & \\
\hline \multicolumn{6}{|l|}{ "B" symptoms } \\
\hline Present & 42 & 33 & 43 & 19 & .003 \\
\hline Absent & 84 & 67 & 186 & 81 & \\
\hline Treatment type & & & & & $<.001$ \\
\hline Radiotherapy only & 2 & 2 & 60 & 26 & \\
\hline Radiotherapy and chemotherapy & 124 & 98 & 169 & 74 & \\
\hline Type of chemotherapy & & & & & $<.001$ \\
\hline Without alkylating agents & 8 & 6 & 93 & 55 & \\
\hline Containing alkylating agents & 116 & 94 & 76 & 45 & \\
\hline Alkylating chemotherapy* & & & & & $<.001$ \\
\hline 1.5 cycles & 14 & 12 & 29 & 38 & \\
\hline Two cycles & 40 & 35 & 27 & 36 & \\
\hline Three cycles & 48 & 41 & 16 & 21 & \\
\hline Six cycles & 14 & 12 & 4 & 5 & \\
\hline
\end{tabular}

$49 \%$ ), $60 \%$ (95\% CI, $47 \%$ to $72 \%$ ), $75 \%$ (95\% CI, $63 \%$ to $85 \%$ ), and $78 \%$ (95\% CI, 52\% to 94\%), respectively $(P<.001)$.

According to the EORTC protocols, all patients older than 50 years were considered unfavorable and received alkylating chemotherapy and radiotherapy. Therefore, age might not be an independent risk factor. A stepwise logistic regression analysis was made in which age, B symptoms, clinical stage, and type of treatment were included as explanatory variables. Two analyses were made. In the first one, the treatment actually received was categorized as radiotherapy only, chemoradiotherapy not including alkylating agents, and chemoradiotherapy including alkylating agents. In the second analysis, the amount of MOPP equivalents was introduced in the regression model. The first model showed that age over 50 years (odds ratio $[\mathrm{OR}]=3.66 ; 95 \% \mathrm{CI}, 1.42$ to $9.47 ; P=.007$ ), stage II disease $(\mathrm{OR}=2.32 ; 95 \% \mathrm{CI}, 1.28$ to $4.19 ; P=.005)$, and the administration of alkylating chemotherapy $(\mathrm{OR}=22.59 ; 95 \% \mathrm{CI}$, 11.12 to $45.89 ; P<.001$ ) were independent factors predicting for post-treatment elevated FSH. When the number of MOPPequivalent cycles of alkylating chemotherapy was introduced in the model, age over 50 years $(\mathrm{OR}=4.37 ; 95 \% \mathrm{CI}, 1.83$ to $10.46 ; P=$ .001 ), 2.0 or $2.5 \mathrm{MOPP}$ equivalents ( $\mathrm{OR}=3.49$; $95 \% \mathrm{CI}, 1.93$ to $6.30 ; P<.001)$ and three to six MOPP equivalents $(\mathrm{OR}=10.85$; $95 \%$ CI, 3.44 to $34.19 ; P<.001$ ) remained statistically significant, supporting the assumption that age did have an independent influence on FSH values and chances of recovery.

\section{Recovery of Fertility (Selection B)}

The treatment actually administered to patients with at least one FSH evaluation 0 to 9 months after treatment is displayed in
Table 2. Time between end of treatment and FSH evaluation for the 101 patients $(29 \%)$ in whom FSH recovery was studied (posttreatment elevated FSH and further evaluations) ranged between 11 and 119 months, with a median of 26 months. According to treatment groups, median time was 27 months for patients treated with alkylating chemotherapy, 18 months for those treated with chemotherapy without alkylators, and 20 months for patients with radiotherapy only.

At first evaluation (median time, 3 months after end of treatment), 55\% of patients (191 of 349) had elevated FSH. Recovery occurred in $42 \%$ ( 42 of 101) at a median time of 23 months (range, 10 to 67 months) after end of treatment (Fig 2). It was 24 months (range, 14 to 67 months) for patients treated with alkylating chemotherapy, 16 months (range, 10 to 39 months) for those treated with chemotherapy without alkylators, and 18 months (range, 10 to 34 months) for patients with radiotherapy only $(P<.001)$. The cumulative probability of gonadal function recovery by treatment type is illustrated in Figure $3 \mathrm{~A}$, where a statistically significant $(P<.001)$ trend was noticed. Besides treatment, no factors influenced recovery.

The influence of the amount of MOPP equivalents was also studied. Among the 79 patients treated with alkylating chemotherapy, 20 received 1.5 MOPP equivalents, 24 received two MOPP equivalents, 23 received three MOPP equivalents and 12 patients received six MOPP equivalents. The cumulative probability of gonadal function recovery by treatment intensity is illustrated in Figure 3B, in which patients $(\mathrm{n}=22)$ treated without alkylators, as well as patients $(\mathrm{n}=20)$ who received one or 1.5 MOPP equivalents and those $(\mathrm{n}=59)$ who received two to six MOPP-equivalent cycles, were 


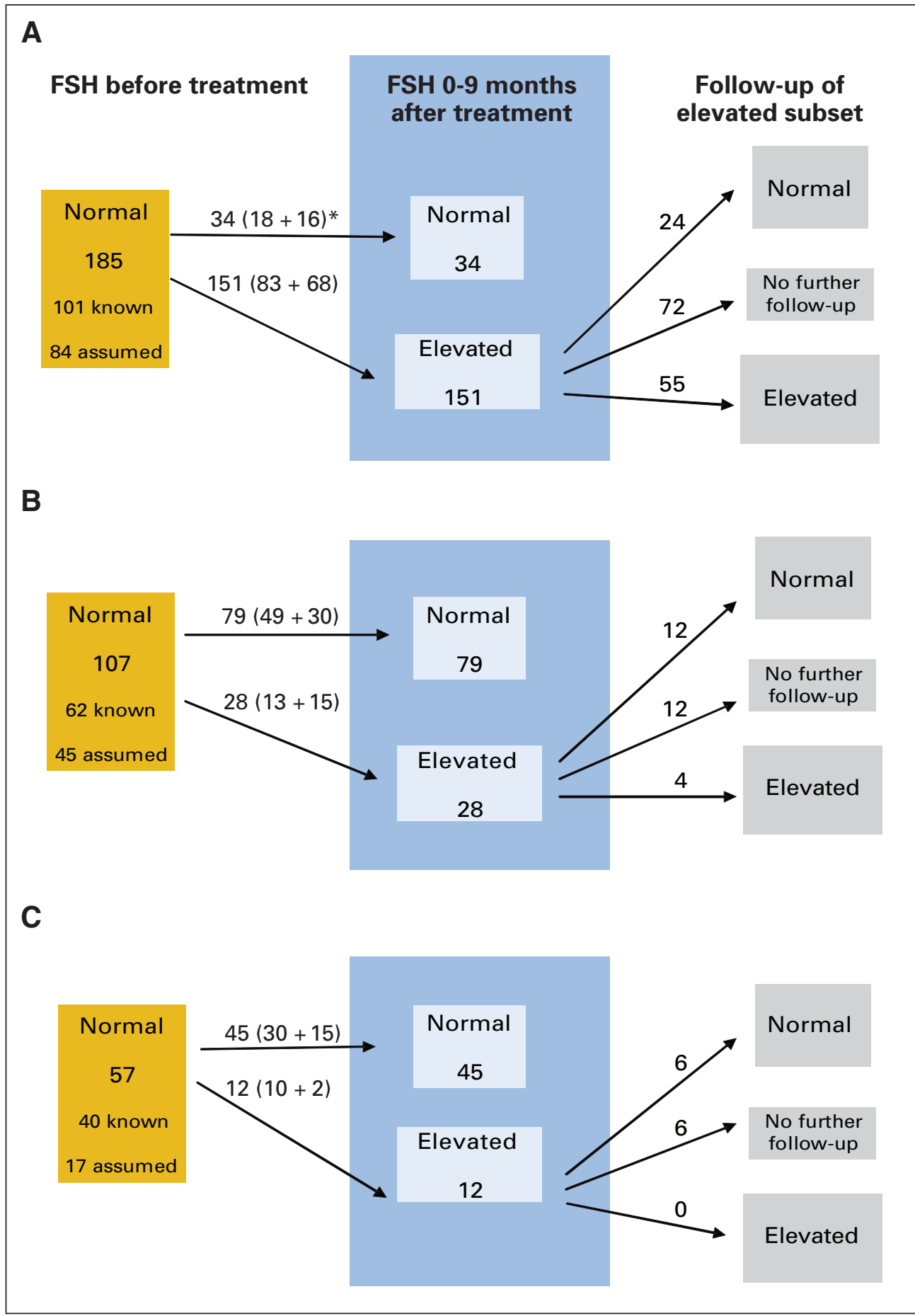

Fig 2. Follicle-stimulating hormone (FSH) analysis per treatment category (Selection B). Left part shows data before start of treatment, middle part shows the early 0 to 9 months after treatment. FSH data and right boxes show the outcome of patients who experienced an elevated FSH between 0 and 9 months. (A) FSH data after alkylating chemotherapy. (B) FSH data after nonalkylating chemotherapy. (C) FSH data after radiotherapy only. (*) Numbers in parenthesis are known plus assumed.

grouped. The three curves differed significantly $(P<.001)$. No other factors were associated with recovery.

\section{DISCUSSION}

In a large cohort of patients with early-stage HL participating in the EORTC (H6-H7) and combined EORTC-GELA trials H8-H9 in whom FSH values were prospectively collected, we confirm that alky- lating chemotherapy has a marked effect on male fertility. Persistent damage was found four times more often than with other treatments. Recovery, when occurring, also took longer. Besides treatment with alkylating agents, factors increasing susceptibility to treatment-related fertility damage were clinical stage II and age over 50 years at start of therapy. The number of cycles of alkylating chemotherapy had a large impact on the outcome of FSH when elevated after treatment in limiting the chances and speed of recovery. 


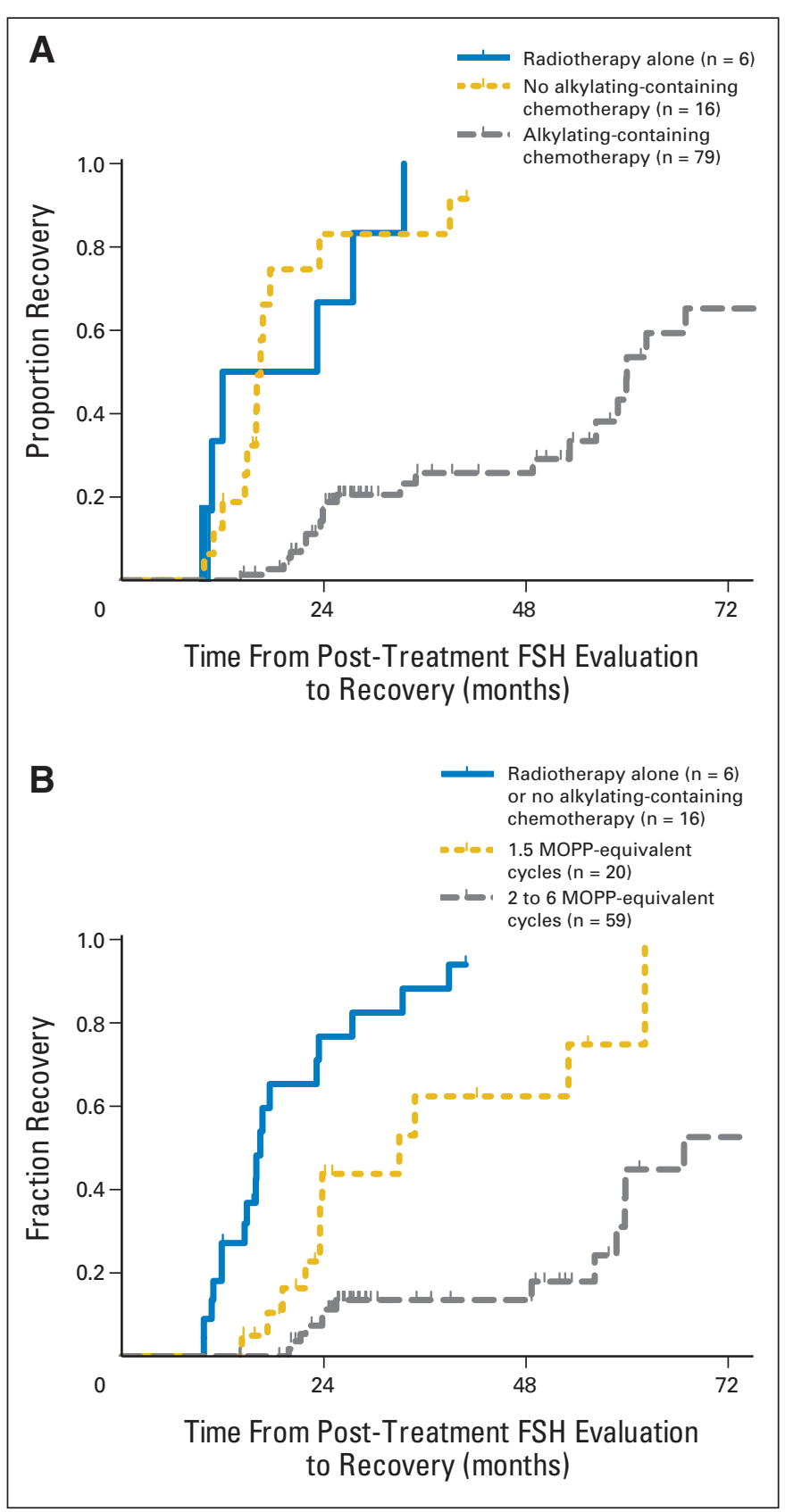

Fig 3. (A) Cumulative probability of gonadal function recovery by treatment type: radiotherapy alone $(n=6)$, radiochemotherapy, and chemotherapy without alkylating agents ( $n=16$ ), radiotherapy and alkylating chemotherapy ( $n=79$ ). The 3-year cumulative probability was 100\% (95\% Cl, 54\% to 100\%), 83\% (95\% $\mathrm{Cl}, 58 \%$ to $97 \%)$, and $26 \%(95 \% \mathrm{Cl}, 16 \%$ to $39 \%)$, respectively (overall $\mathrm{P}<.001)$. (B) Cumulative probability of gonadal function recovery by treatment intensity: radiotherapy alone or (radio-)chemotherapy without alkylating agents ( $n=22$ ), radiotherapy and alkylating chemotherapy including 1.5 mechlorethamine, vincristine, procarbazine, and prednisone (MOPP)-equivalent cycles $(n=20)$ or two to six MOPP-equivalent cycles $(n=59)$. The 3-year cumulative probability was $89 \%(95 \% \mathrm{Cl}, 70 \%$ to $98 \%), 63 \%(95 \% \mathrm{Cl}, 38 \%$ to $87 \%)$ and $14 \%(95 \% \mathrm{Cl}, 7 \%$ to $26 \%)$, respectively (overall $P<.001)$. $\mathrm{FSH}$, folliclestimulating hormone.

Our results are consistent with those of Viviani et al, Marmor and Duyck, and Specht et al, who mainly studied MOPP chemotherapy. $^{24-26}$ Although the ABVD regimen-among others-was developed because of its fertility-sparing capacity (which we con- firm hereby), the MOPP-ABV regimen and especially the newer BEACOPP regimen appear to represent a step back in this regard.

Our data provide additional information on recovery of fertility and time before recovery. Using a subset of patients with FSH evaluations both during the 0 to 9 month period after treatment completion and later on, we show that immediately after alkylating chemotherapy, FSH was elevated in a large majority of patients, irrespectively of the number of MOPP-equivalent cycles administered. However, time to recovery, if any, was proportional to the number of cycles of alkylating chemotherapy. These results are consistent with data obtained by Da Cunha et al. ${ }^{7}$

Radiotherapy also has an influence on fertility. ${ }^{27}$ Patients included in this study have received either mantle-field irradiation, IF-RT (always supradiaphragmatic) or subtotal nodal irradiation, the latter extending to the level of the fourth lumbar vertebra. Scatter irradiation from subdiaphragmatic radiotherapy can possibly cause gonadal damage. ${ }^{4}$ However, scatter irradiation from the para-aortic fields is estimated to be around $1 \% .^{28}$ In our study, STNI had a small effect on FSH: Patients made a fast and complete recovery, and at a median time of 31 months after treatment, only 3\% maintained an elevated FSH. These results are in agreement with those of Shapiro et $\mathrm{al}^{29}$ and Kinsella et al. ${ }^{30}$

Our results are based on a large group of patients with clinical stage I or II HL representative of those included in the last four trials conducted by the EORTC Lymphoma Group and the GELA. Furthermore, they concern data on recent chemotherapy schedules. They highlight the risk of infertility experienced by male patients treated with BEACOPP if this chemotherapy is preferred to ABVD as standard treatment. One can also hypothesize that with escalated BEACOPP, damage to fertility will be even higher. However, intensive chemotherapy regimens such as escalated BEACOPP could be the preferable treatment in patients with an unfavorable risk profile, such as patients with early positron emission tomography positivity or patients with three or more adverse factors. ${ }^{31}$ For them, the balance between avoidance of toxic therapy and the risk of relapse-inevitably followed by much more toxic salvage therapy_clearly points to the selection of the most effective initial therapy even if fertility cannot be spared.

In this study, potential sources of bias can have occurred. Firstly, fertility could have been evaluated more often in patients at risk (ie, in those treated with alkylating chemotherapy). However, the proportions of patients included in this study were distributed equally among the various treatment groups (Table 1). Secondly, the number of post-treatment FSH evaluations and the time interval could have been larger in patients with earlier abnormal FSH values. Again, this is not the case because the occurrence and the number of evaluations appeared independent of pretreatment FSH values.

In conclusion, we demonstrate in the largest series studied so far that male fertility can be secured after chemotherapy for HL if alkylating agents are excluded. Even if an elevated FSH is observed within the first months after the end of such chemotherapy, recovery is frequently seen. In contrast, full-dose alkylating chemotherapy is associated with dismal prognosis for the majority of patients. Even if only a limited number of cycles are administered, recovery is incomplete in many patients. Finally, age above 50 years and stage II disease contribute to a poor outcome as far as fertility and recovery are concerned. 


\section{AUTHORS' DISCLOSURES OF POTENTIAL CONFLICTS} OF INTEREST

The author(s) indicated no potential conflicts of interest.

\section{AUTHOR CONTRIBUTIONS}

Conception and design: Marleen A.E. van der Kaaij, John M.M. Raemaekers, Hanneke C. Kluin-Nelemans, Michel Henry-Amar Financial support: John M.M. Raemaekers, Hanneke C.

Kluin-Nelemans, Michel Henry-Amar

Administrative support: Hanneke C. Kluin-Nelemans, Michel Henry-Amar Provision of study materials or patients: John M.M. Raemaekers, Patrice Carde, Evert M. Noordijk, Christophe Fermé, José Thomas,
Houchingue Eghbali, Hanneke C. Kluin-Nelemans, Michel

Henry-Amar

Collection and assembly of data: Marleen A.E. van der Kaaij, Nolwenn Le Stang, Michel Henry-Amar

Data analysis and interpretation: Marleen A.E. van der Kaaij, Natacha Heutte, Arnold H.M. Simons, Hanneke C. Kluin-Nelemans, Michel Henry-Amar

Manuscript writing: Marleen A.E. van der Kaaij, John M.M. Raemaekers, Arnold H.M. Simons, Houchingue Eghbali, Hanneke C. Kluin-Nelemans, Michel Henry-Amar

Final approval of manuscript: Marleen A.E. van der Kaaij, Natacha Heutte, Nolwenn Le Stang, John M.M. Raemaekers, Arnold H.M. Simons, Patrice Carde, Evert M. Noordijk, Christophe Fermé, José Thomas, Houchingue Eghbali, Hanneke C. Kluin-Nelemans, Michel Henry-Amar

\section{REFERENGES}

1. Sieber M, Rueffer $U$, Josting $A$, et al: Treatment of Hodgkin's disease: Current strategies of the German Hodgkin's Lymphoma Study Group. Ann Oncol 10:23-29, 1999 (suppl 6)

2. Raemaekers J, Kluin-Nelemans $\mathrm{H}$, Teodorovic I, et al: The achievements of the EORTC Lymphoma Group: European Organisation for Research and Treatment of Cancer. Eur J Cancer 38:S107-S113, 2002 (suppl 4)

3. Chen YT, Zheng T, Chou MC, et al: The increase of Hodgkin's disease incidence among young adults: Experience in Connecticut, 19351992. Cancer 79:2209-2218, 1997

4. Howell SJ, Shalet SM: Spermatogenesis after cancer treatment: Damage and recovery. J Natl Cancer Inst Monogr 12-17, 2005

5. Grigg A: The impact of conventional and highdose therapy for lymphoma on fertility. Clin Lymphoma 5:84-88, 2004

6. Blumenfeld Z, Dann E, Avivi I, et al: Fertility after treatment for Hodgkin's disease. Ann Oncol 13:138-147, 2002 (suppl 1)

7. da Cunha MF, Meistrich ML, Fuller LM, et al: Recovery of spermatogenesis after treatment for Hodgkin's disease: Limiting dose of MOPP chemotherapy. J Clin Oncol 2:571-577, 1984

8. Anselmo AP, Cartoni $C$, Bellantuono $P$, et al Risk of infertility in patients with Hodgkin's disease treated with $A B V D$ vs MOPP vs ABVD/MOPP. Haematologica 75:155-158, 1990

9. Viviani S, Ragni G, Santoro A, et al: Testicular dysfunction in Hodgkin's disease before and after treatment. Eur J Cancer 27:1389-1392, 1991

10. Canellos GP, Anderson JR, Propert KJ, et al: Chemotherapy of advanced Hodgkin's disease with MOPP, ABVD, or MOPP alternating with ABVD. N Engl J Med 327:1478-1484, 1992

11. Carde $P$, Hagenbeek $A$, Hayat $M$, et al: Clinical staging versus laparotomy and combined modality with MOPP versus ABVD in early-stage Hodgkin's disease: The $\mathrm{H} 6$ twin randomized trials from the European Organization for Research and Treatment of Cancer Lymphoma Cooperative Group. J Clin Oncol 11:2258-2272, 1993

12. DeVita VT Jr, Serpick AA, Carbone PP: Combination chemotherapy in the treatment of advanced Hodgkin's disease. Ann Intern Med 73:881-895, 1970

13. Bonadonna G, Zucali R, Monfardini S, et al: Combination chemotherapy of Hodgkin's disease with adriamycin, bleomycin, vinblastine, and imidazole carboxamide versus MOPP. Cancer 36:252 259, 1975

14. Santoro A, Bonfante V, Bonadonna G: Salvage chemotherapy with $A B V D$ in MOPP-resistant Hodgkin's disease. Ann Intern Med 96:139-143, 1982

15. Noordijk EM, Carde P, Dupouy N, et al: Combined modality therapy for all patients with clinical stage I-II Hodgkin's lymphoma. Long term results of the EORTC H7 randomized controlled trials (19881993). J Clin Oncol 24:3128-3135, 2006

16. Zittoun $R$, Eghbali $H$, Audebert $A$, et al: The combination of epirubicin, bleomycin, vinblastine and prednisone (EBVP) before radiotherapy in localized stages of Hodgkin's disease. Phase II trials [French]. Bull Cancer 74:151-157, 1987

17. Klimo P, Connors JM: MOPP/ABV hybrid program: Combination chemotherapy based on early introduction of seven effective drugs for advanced Hodgkin's disease. J Clin Oncol 3:1174-1182, 1985

18. Ferme $\mathrm{C}$, Eghbali $\mathrm{H}$, Meerwaldt $\mathrm{JH}$, et al: Combined Modality Treatment for Early Stages Hodgkin's Lymphoma Based on Prognostic Factors. Results of the EORTC-GELA H8 Trial. N Engl J Med (in press)

19. Noordijk EM, Thomas J, Ferme C, et al: First results of the EORTC-GELA H9 randomized trials: The H9-F trial (comparing 3 radiation dose levels) and H9-U trial (comparing 3 chemotherapy schemes) in patients with favorable or unfavorable early stage Hodgkin's lymphoma (HL). J Clin Oncol 23:561s, 2005 (suppl; abstr 6505)

20. Ferme $C$, Divine $M$, Vranovsky A, et al: Four $A B V D$ and involved-field radiotherapy in unfavorable supradiaphragmatic clinical stages (CS) I-| Hodgkin's lymphoma (HL): Preliminary results of the EORTC-GELA H9-U trial. Proc Am Soc Hematol 106:813, 2005 (abstr)

21. Eghbali $H$, Brice $P$, Creemers $G Y$, et al: Comparison of three radiation dose levels after EBVP regimen in favorable supradiaphragmatic clinical stages (CS) I-II Hodgkin's lymphoma (HL): Preliminary results of the EORTC-GELA H9-F trial. Proc Am Soc Hematol 106:814, 2005 (abstr)

22. Diehl V, Sieber M, Ruffer U, et al: BEACOPP. An intensified chemotherapy regimen in advanced Hodgkin's disease: The German Hodgkin's Lymphoma Study Group. Ann Oncol 8:143-148, 1997

23. Rothman K, Boice J: Epidemiologic Analysis With a Programmable Calculator. Boston, MA, Epidemiology Resources Inc, 1982

24. Viviani S, Santoro A, Ragni G, et al: Gonadal toxicity after combination chemotherapy for Hodgkin's disease: Comparative results of MOPP vs ABVD. Eur $J$ Cancer Clin Oncol 21:601-605, 1985

25. Marmor D, Duyck F: Male reproductive potential after MOPP therapy for Hodgkin's disease: A long-term survey. Andrologia 27:99-106, 1995

26. Specht $L$, Geisler $C$, Hansen MM, et al: Testicular function in young men in long-term remission after treatment for the early stages of Hodgkin's disease. Scand J Haematol 33:356-362, 1984

27. Rowley MJ, Leach DR, Warner GA, et al: Effect of graded doses of ionizing radiation on the human testis. Radiat Res 59:665-678, 1974

28. Fraass $B A$, van de Geÿn J: Peripheral dose from megavolt beams. Med Phys 10:809-818, 1983

29. Shapiro E, Kinsella TJ, Makuch RW, et al: Effects of fractionated irradiation of endocrine aspects of testicular function. J Clin Oncol 3:1232 1239, 1985

30. Kinsella TJ, Trivette G, Rowland J, et al: Long-term follow-up of testicular function following radiation therapy for early-stage Hodgkin's disease. J Clin Oncol 7:718-724, 1989

31. Hasenclever D, Diehl V: A prognostic score for advanced Hodgkin's disease: International Prognostic Factors Project on Advanced Hodgkin's Disease. N Engl J Med 339:1506-1514, 1998 